\title{
Discovery of Warm Gas in the Virgo Cluster
}

\author{
R. LIEU, ${ }^{1}$ J. P. D. MITTAZ, ${ }^{2}$ S. BOWYER, ${ }^{1}$ \\ J. H. M. M. SCHMITT, ${ }^{1,3}$ AND J. LEWIS ${ }^{1}$ \\ ${ }^{1}$ Center for EUV Astrophysics, 2150 Kittredge Street, \\ University of California, Berkeley, CA $94720-5030$, USA
}

\author{
${ }^{2}$ Mullard Space Science Laboratory, Holmbury St. Mary, Dorking, Surrey RH5 6NT, UK \\ ${ }^{3}$ Max Planck Institut für Extraterrestrische Physik, W-8046 Garching-bei-München, Germany
}

During the EUVE sky survey of the Virgo region, a central source positionally coincident with the X-ray emitting galaxy M87, and a surrounding halo of extended emission, were detected in the $0.065-0.248 \mathrm{keV}$ band. A detailed comparison of these data with the ROSAT PSPC data of M87 revealed an excess flux at energies $<0.4 \mathrm{keV}$ within the central $30^{\prime}$ radius which cannot be associated with the well-known cluster gas at $\mathrm{X}$-ray temperatures ( $\mathrm{kT} \geq \mathrm{a}$ few keV). Instead, it is necessary to introduce a second gas component, of temperature $T \sim 5 \times 10^{6} K(\mathrm{kT} \sim 0.1$ $\mathrm{keV}$ ). The resulting two-component model (warm + hot) can account for all the data. The origin and stability of the warm component, with a temperature near the peak of the thermal plasma cooling curve, is unclear. Both the temperature and spatial extent argue against cooling flow as the primary process responsible for its production. Other mechanisms, such as a galactic wind and heating by galaxy motions, must be considered.

From soft X-ray $(\hbar \omega \sim 3 \mathrm{keV})$ data gathered by the EINSTEIN, EXOSAT and ROSAT missions, it is well known that M87, the central galaxy of the Virgo cluster, has a halo of emission extending to a radius of $100^{\prime}$ (Fabricant \& Gorenstein 1983; Stewart et al. 1984; Forman, Jones \& Binggeli 1985; Böhringer et al. 1994) and that a cooling flow exists in the innermost $20^{\prime}$ region. We present new observational results of M87 obtained from $E U V E$ and combine these results with data from the ROSAT mission for our analysis. The source was detected by the EUVE sky survey in the Lex/B (65-248 eV) filter passband, with an exposure of approximately $1,000 \mathrm{~s}$. Figure 1 is a radial profile of the Lex/B count rates. The data are expressed in units of surface brightness of diffuse emission (i.e., counts $\mathrm{ks}^{-1} \operatorname{arcmin}^{-2}$ ); they are average values for concentric annuli moving outwards from the best-fit position of a central source, which is approximately $1^{\prime}$ offset from the optical position of M87. The background at large radii $\left(>35^{\prime}\right)$ is flat, so that a sizable region is available for its determination with high accuracy. The brightness distribution within a radius of $5^{\prime}$ is consistent with a point source detected at $4.2 \sigma$ significance. More surprisingly, an independent halo of extended emission was detected at $3.3 \sigma$ significance between $5^{\prime}$ and $35^{\prime}$ radii. These data represent the first reported detection of extended cluster emission in the EUV.

In order to compare the $E U V E$ data with existing X-ray data of M87, we extracted from the public archive the results of a ROSAT PSPC pointed observation which took place in July 1992 , with an exposure of nearly $10,000 \mathrm{~s}$. The analysis was performed with the STARLINK ASTERIX X-ray data processing system. Periods of high background and poor attitude solutions were removed from the data, and the pulse-height spectra were computed for concentric annuli centered at the $\mathrm{X}$-ray position of M87. We only considered energies $\hbar \omega>0.18 \mathrm{keV}$, and avoided the region between $19^{\prime}$ and $22^{\prime}$ to minimize the effects of the support structure on the analysis. The extracted spectra were also corrected for vignetting and dead times. An accurate model for the local soft 


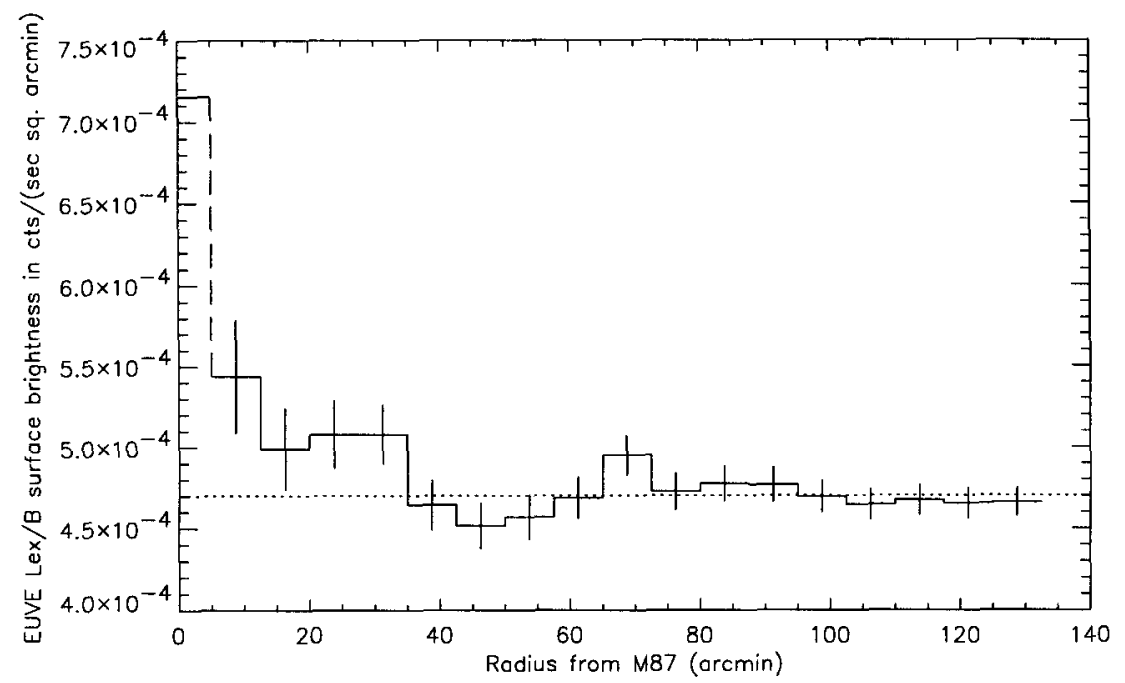

FIGURE 1. EUVE Lex/B (0.065-0.248 keV) filter count rates gathered during the sky survey are expressed in units of diffuse emission for concentric annuli centered at the best-fit position of a point source, which is offset from the optical position of M87 by $544^{\prime \prime} 2$. The innermost $5^{\prime}$ region (i.e., first data point) corresponds to this point source, detected at $4.2 \sigma$ significance by the standard source search procedure of matched-filtering. The region between $5^{\prime}$ and $35^{\prime}$ corresponds to a diffuse excess which is $3.3 \sigma$ above a background (dotted line) determined by using all the data in the "flat" region beyond 35 '.

$\mathrm{X}$-ray diffuse background was developed, essentially by fitting the $1 / 4 \mathrm{keV}$-band spectrum for the $45^{\prime}-57^{\prime}$ region with the thin plasma emission code of Mewe et al. $(1985,1986)$, assuming a foreground absorption corresponding to a $\mathrm{HI}$ column density of $5.0 \times 10^{18}$ $\mathrm{cm}^{-2}$ to account for the local solar cloud. This background model is a constant additive component used in the interpretation of data for all the inner annuli, after the model has been normalized to the appropriate sky areas of each annulus, taking into account obstruction by the detector wires and spokes. We also consulted the master-veto data to ensure that the particle background is negligible. Owing to the radial dependence of the emission properties, the PSPC spectra for concentric annuli extending out to a radius of $45^{\prime}$ were individually fitted with the thin plasma emission code of Mewe et al., using the appropriate off-axis response matrix. Interstellar absorption corresponding to a Galactic H I column density of $2.5 \times 10^{20} \mathrm{~cm}^{-2}$ (Stark et al. 1992) was assumed. A redshift of $z=0.0043$ was also assumed.

We find that the EUVE and ROSAT data for the central ring of inner radius $5^{\prime}$ and outer radius $35^{\prime}$ (i.e., excluding the emission from M87) cannot simultaneously be fitted with a single temperature plasma model. While the $\mathrm{X}$-ray temperature plasma can account for most of the PSPC count rates at energies $\hbar \omega>0.4 \mathrm{keV}$, it does not produce sufficient EUV radiation to match the observed Lex/B count rates. Instead, it is necessary to introduce a second gas component of considerably lower temperature ( $\mathrm{kT}$ $\sim 0.1 \mathrm{keV}$ ). Predictions of the resultant two-phase model agree well with the data in both passbands. They are listed in Table 1.

As we study the new emission component in more detail, we find that no single tem- 
TABLE 1. M87 Halo between $5^{\prime}$ and $35^{\prime}$

$\begin{array}{lcc} & \begin{array}{c}\text { EUVE counts ks } \\ (0.065-0.25 \mathrm{keV})\end{array} & \begin{array}{c}\text { ROSAT X-ray counts s } \\ (0.4-2.0 \mathrm{keV})\end{array} \\ \text { Observed } & 150.8 \pm 45.2 & 18.97 \pm 0.04 \\ \text { Warm Plasma Model } & 35.0 & 0.2 \\ \begin{array}{l}\text { Hot Plasma Model } \\ \text { Hot }\end{array} & 57.7 & 18.67 \\ T=2.76 \times 10^{5} \mathrm{~K} & & \\ \text { Total } & & 18.87 \\ \text { (two-phase model) } & 92.7 & \end{array}$

perature gas scenario can explain the PSPC spectrum of an inner annulus for the entire range of energies. Data at energies $\hbar \omega>0.4 \mathrm{keV}$ are satisfactorily fitted by a single temperature plasma model of Mewe et al., with $\mathrm{kT} \sim$ a few $\mathrm{keV}$. At energies $\hbar \omega<0.4$ $\mathrm{keV}$, however, there exists a "soft excess" in the spectra. This is illustrated in Figure 2 where we show the spectrum and folded model for a typical inner annulus, of mean radius $12 \prime 5$ and width 2.5 . The dotted line represents the best-fit model for the $>0.4 \mathrm{keV}$ band, which falls short of the data points at energies $<0.4 \mathrm{keV}$. It is unlikely for such a "soft excess" to be caused by systematic errors in the $1 / 4 \mathrm{keV}$-band calibration of the PSPC, because an extensive analysis of this band by Snowden et al. (1995) demonstrated that the published effective areas are overestimated by only $10 \%$. Moreover, we find that the surface brightness of soft excess is peaked at the center of the cluster. This correlation strongly suggests that the excess is a real effect related to the M87 emission, rather than to any extraneous contamination not removed by the background subtraction procedure.

We find that the full PSPC spectrum for the $12.5 \pm 2.5$ annulus can be fitted by a two-temperature plasma with common metallicity and with a second component having $\mathrm{kT} \sim 0.083 \mathrm{keV}\left(T \sim 9.6 \times 10^{5} \mathrm{~K}\right)$, which we call the "warm component." The solid line in Figure 2 shows the best-fit model. For all other annuli within a radius of $35^{\prime}$ equally good fits can be obtained by the use of two-temperatures. However, spectra taken outside $35^{\prime}$ can be fitted by a single temperature plasma, and moreover there is no detected Lex/B flux in this region. Although the PSPC does not have the spectral resolution to separate the different temperatures of the emitting material at various radii along the observational line-of-sight, it is the existence of much cooler plasmas at the inner region which characterizes the new result presented here.

The PSPC data have sufficient statistical quality to enable a comparison of the properties of the two gas components in the cluster. In Figures 3 and 4 we plot the radial temperature profiles of the hot and warm plasmas. It is clear that the hot component cools inside the central $20^{\prime}$ region. Within the accuracy of the data, there is no evidence of similar behavior in the warm plasma. In Figures 5 and 6 we plot the radial surface brightness profiles of the two plasmas, to demonstrate that the emission from the hot gas does not fall as rapidly w.r.t. radius. The hot component remains detectable beyond the radius of $35^{\prime}$ where the warm component can no longer be found. The best-fit common metallicity varies between 0.37 and 0.56 of the solar abundance, with a trend of higher 


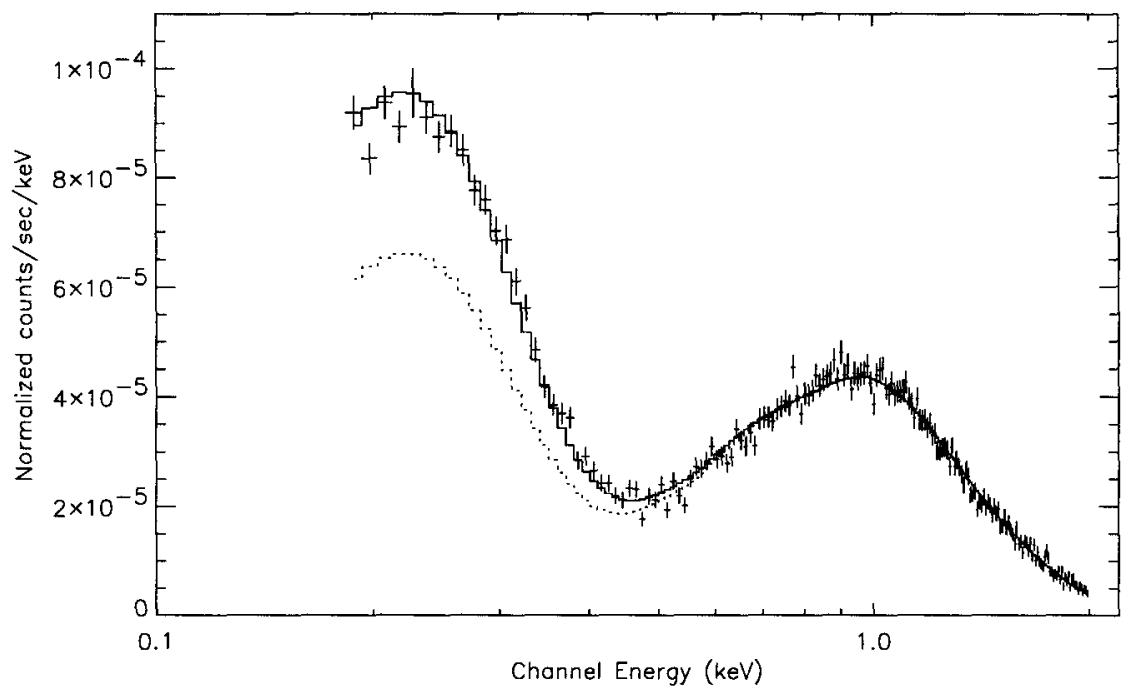

FIGURE 2. Data and folded model of the ROSAT PSPC spectrum for an annulus centered at $\mathrm{M} 87$, of mean radius 12.5 and width 2.5 . Dotted line gives the best-fit single-temperature Mewe thin plasma emission model for the $>0.4 \mathrm{keV}$ data, while solid line gives the best-fit two-temperature model for the entire spectral range. This model includes a "warm" plasma as second component.

metal content at smaller radii, consistent with results of the PSPC sky survey (Böhringer et al. 1994).

An important question concerning the newly detected gas component has to do with its maintenance, because an optically thin plasma at temperature $\mathrm{kT}<0.1 \mathrm{keV}$ cools rapidly by line emissions. Using the best-fit parameter values for the $31^{\prime} \pm 4^{\prime}$ (i.e., limiting) annulus, we estimated the plasma density to be $n_{e} \sim 4.73 \times 10^{-4} \mathrm{~cm}^{-3}$ and the cooling time $\tau \sim 3.16 \times 10^{8}$ years, assuming that the warm plasma fills the entire emission volume. Since the cooling time is much less than the age of the cluster, a mechanism of sustaining the gas is necessary, irrespective of its origin. The mass of warm gas within the same volume is estimated to be $7.92 \times 10^{10} M_{\odot}$.

We argue that the warm gas is unlikely to be associated with mass deposition throughout the cooling flow, because (a) given that the hot gas cools from $\mathrm{kT} \sim 3 \mathrm{keV}$ at $20^{\prime}$ radius to $\mathrm{kT} \sim 1 \mathrm{keV}$ at the center, it is difficult to envisage the deposition of $\mathrm{kT} \sim$ $0.1 \mathrm{keV}$ gas everywhere, unless the gas is extremely inhomogeneous; (b) there is no observational evidence of cooled material - the published result on the presence of cold absorbing matter in the center of M87 (White et al. 1991) is inconsistent with the data reported here, since our models of the PSPC spectra do not involve $N_{H}$ values as high as $10^{21} \mathrm{~cm}^{-2}$; (c) if the warm gas is continuously replenished by cooling flow as it rapidly leaves the peak of the cooling function to form cold material, we estimate the mass of such material to be $\sim 10^{13} M_{\odot}$ in the central region; this would provide very significant absorption to the EUV and X-ray photons, which is not observed. 


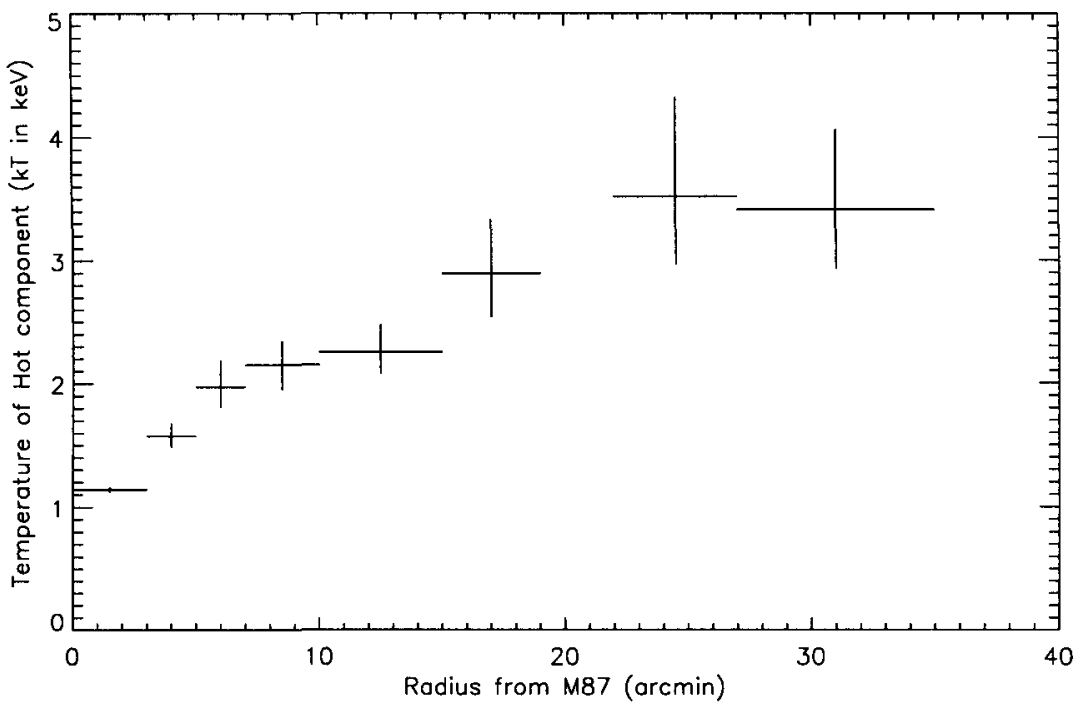

FIGURE 3. Radial profile of the best-fit hot component temperature

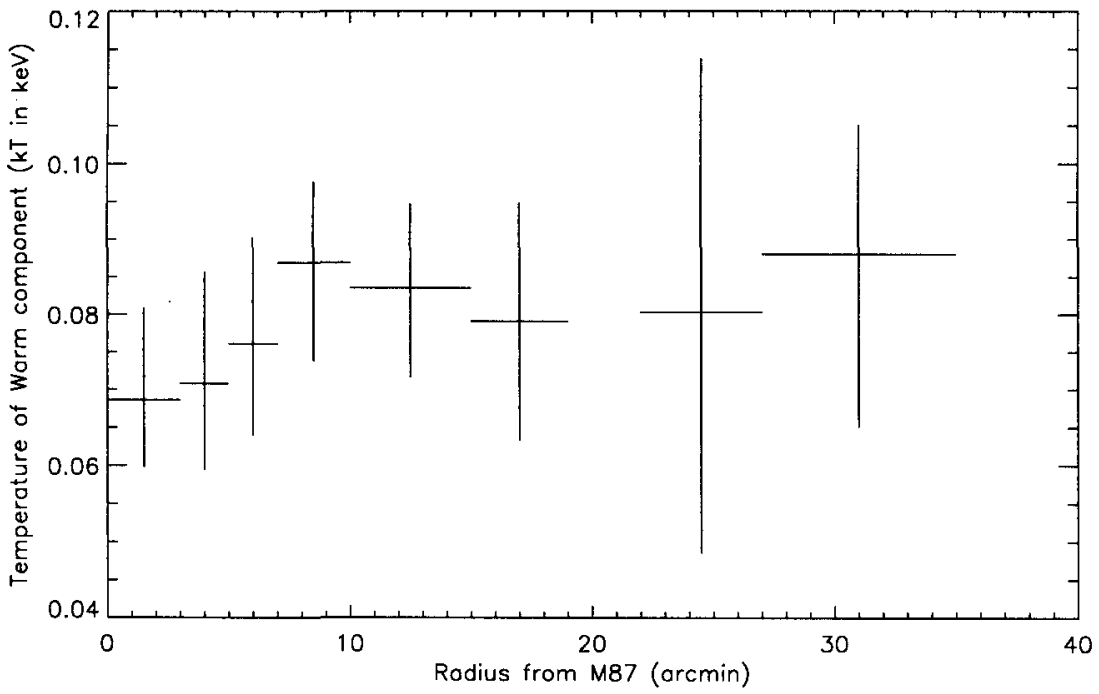

FIGURE 4. Radial profile of the best-fit warm component temperature

We conclude by suggesting an alternative mechanism, viz. a galactic wind of $10^{6} \mathrm{~K}$ plasma (responsible for the soft X-ray background) driven out of the galaxies by cosmic ray pressure (Axford 1981), and which may further be heated as the bulk flow energy due to the motion of the galaxy w.r.t. the cluster is thermalized by collisions. The intracluster gas itself could also be heated through viscous forces between the gas and the moving galaxies (Sarazin 1986). 


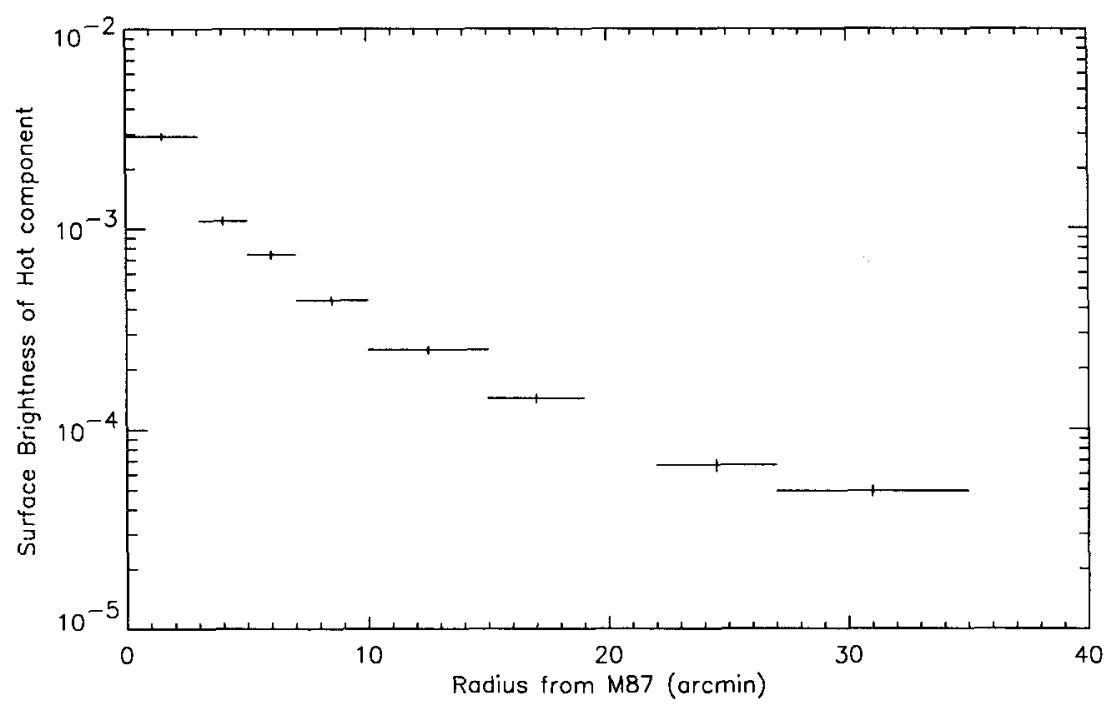

Figure 5. Radial profile of the best-fit surface brightness of the hot component. This surface brightness is related to the emission measure $\left(\mathrm{EM}\right.$, in $\left.\mathrm{cm}^{-3}\right)$ by $\mathrm{EM}=4.77 \times 10^{66} \times$ norm. $\times$ (area of annulus in $\operatorname{arcmin}^{2}$ )

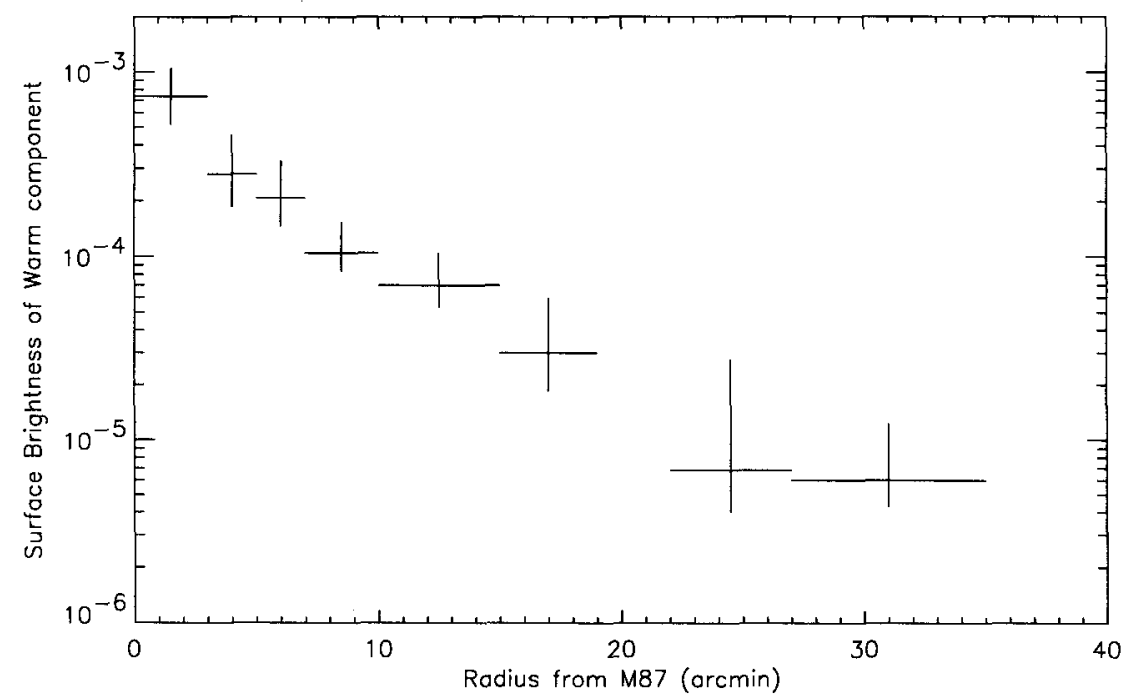

FIGURE 6. Radial profile of the best-fit surface brightness of the warm component, defined in the same way as in Fig. 5. 
We thank R. Stern and D. Leahy for helpful discussions. R. Lieu, S. Bowyer and J. Lewis acknowledge the support of NASA contract NAS5-30180.

\section{REFERENCES}

Axford, W. I. 1981, 17th International Cosmic Ray Conference, 12, 155

Böhringer, H., Briel, U. G., Schwarz, R. A., Voges, W., Hartner, G. \& Trümper, J. 1994, Nature, 368,828

FABRicANT, D. \& GoRENSTEIN, P. 1983, ApJ, 267, 535

Forman, W. Jones, C. \& DEFACCIO, M. 1985, ESO Workshop on the Virgo Cluster of Galaxies, ed. Richter, O.-G. \& Binggeli, B., ESO Conf. Proc. No. 20, ESO, Garching, 323 Mewe, R., GronenschIDd, E. H. B. M. \& VAN DEN Oond 1985, A\&AS, 62, 197

Mewe, R., Lemen, J. R. \& VAN DEN OoRD, G. H. J. 1986, A\&AS, 65, 511

SaraziN, C. L. 1986, Rev. Mod. Phys., 58, 1

SNOWDEN, S. ET AL. 1995, ApJ, submitted

Stark, A. A., Gammie, C. F., Wilson, R. W., Bally, J., Linke, R. A., Heiles, C. \& Hurwitz, M. 1992, ApJS, 79, 77

Stewart, G. C., Canizares, C. R., Fabian, A. C. \& Nulsen, P. E. J. 1984, ApJ, 278, 536

White, D. A., Fabian, A. C., Johnstone, R. M., Mushotzky, R. F. \& Arnaud, K. A. 1991, MNRAS, 252, 72 